\title{
Assessment of the utility of a symptom-based algorithm for identifying febrile patients for malaria diagnostic testing in Senegal
}

Julie Thwing ${ }^{1 *}\left(\mathbb{D}\right.$, Fatou Ba ${ }^{2}$, Alou Diaby $^{3}$, Younouss Diedhiou ${ }^{4}$, Assane Sylla ${ }^{3}$, Guelaye Sall ${ }^{3}$, Mame Birame Diouf ${ }^{5}$, Alioune Badara Gueye ${ }^{2}$, Seynabou Gaye², Medoune Ndiop², Moustapha Cisse², Daouda Ndiaye ${ }^{6}$ and Mady $\mathrm{Ba}^{2,7}$

\begin{abstract}
Background: Malaria rapid diagnostic tests (RDTs) enable point-of-care testing to be nearly as sensitive and specific as reference microscopy. The Senegal National Malaria Control Programme introduced RDTs in 2007, along with a case management algorithm for uncomplicated febrile illness, in which the first step stipulates that if a febrile patient of any age has symptoms indicative of febrile illness other than malaria (e.g., cough or rash), they would not be tested for malaria, but treated for the apparent illness and receive an RDT for malaria only if they returned in $48 \mathrm{~h}$ without improvement.

Methods: A year-long study in 16 health posts was conducted to determine the algorithm's capacity to identify patients with Plasmodium falciparum infection identifiable by RDT. Health post personnel enrolled patients of all ages with fever $\left(\geq 37.5^{\circ} \mathrm{C}\right)$ or history of fever in the previous 2 days. After clinical assessment, a nurse staffing the health post determined whether a patient should receive an RDT according to the diagnostic algorithm, but performed an RDT for all enrolled patients.

Results: Over 1 year, 6039 patients were enrolled and 58\% (3483) were determined to require an RDT according to the algorithm. Overall, 23\% (1373/6039) had a positive RDT, 34\% (1130/3376) during rainy season and 9\% (243/2661) during dry season. The first step of the algorithm identified only $78 \%$ of patients with a positive RDT, varying by transmission season (rainy 80\%, dry 70\%), malaria transmission zone (high 75\%, low 95\%), and age group (under 5 years 68\%, 5 years and older 84\%).
\end{abstract}

Conclusions: In all but the lowest malaria transmission zone, use of the algorithm excludes an unacceptably large proportion of patients with malaria from receiving an RDT at their first visit, denying them timely diagnosis and treatment. While the algorithm was adopted within a context of malaria control and scarce resources, with the goal of treating patients with symptomatic malaria, Senegal has now adopted a policy of universal diagnosis of patients with fever or history of fever. In addition, in the current context of malaria elimination, the paradigm of case management needs to shift towards the identification and treatment of all patients with malaria infection.

Keywords: Malaria, Diagnosis, Treatment, Algorithm, Fever, Case management, Rapid diagnostic test

\footnotetext{
*Correspondence: fez3@cdc.gov

1 U.S. Centers for Disease Control and Prevention and President's Malaria

Initiative, Atlanta, USA

Full list of author information is available at the end of the article
} 


\section{Background}

Senegal is a malaria-endemic country in the Sahel zone on the west coast of Africa. Malaria transmission is highly variable across the country, with very low transmission in the north, increasing towards the south. Dakar, the capital city, experiences heterogeneous transmission. Transmission is highly seasonal, with the majority of cases occurring during and just after the rainy season, which generally lasts from July through October. The Senegal National Malaria Control Programme (NMCP) has rapidly scaled up malaria control interventions during the last decade. Artemisinin-based combination therapy (ACT) was introduced in 2006 [1], first at health facilities, and then at community level. Following a feasibility study [2], rapid diagnostic tests (RDTs) were introduced in late 2007, first at health facility level, and then at community level [3]. By 2009, $86 \%$ of suspected malaria cases received a diagnostic test [4], greatly decreasing the use of ACT. Microscopy is used in referral health centres and hospitals and is used almost exclusively for hospitalized patients.

After lengthy consultations with stakeholders, including infectious disease physicians, regional and district medical officers, other Ministry of Health officials, and financial and technical partners, a symptom-based case management algorithm was introduced, along with RDTs, which changed the definition of a suspected malaria case. In 2007, Senegal had just entered the phase of rapid scale-up of malaria control interventions, and the number of cases of fever exceeded available funding needed to test all fever cases. In view of conserving RDTs and testing those most likely to have malaria, the first step of the case management algorithm directed that not all patients with uncomplicated febrile illness or history of fever should receive an RDT. While those without an obvious fever source other than malaria were recommended to receive an RDT, those that also had symptoms or signs indicative of another source of fever (ear drainage, sore throat, sputum, cough, rash, or other fever source, according to the clinical judgment of the healthcare provider) were recommended to be treated for that indication and return for a follow-up visit $48 \mathrm{~h}$ later, at which point if there was no clinical improvement, an RDT was recommended. Patients with positive RDTs were to receive treatment with an ACT (Fig. 1). At community level, all cases of fever continued to be tested. The rationale behind this strategy was that even if some of these patients with fever and symptoms of another fever source did in fact have parasitaemia, it was, in effect, an asymptomatic parasitaemia, with the fever attributable to another source.

As the nation gained experience with the algorithm, concerns grew that the algorithm might miss an unacceptable number of patients with parasitaemia accompanying other symptoms, with dangerous consequences, particularly for children under 5 years old. Health workers in higher transmission zones complained that if they followed the algorithm, they missed patients with malaria. The proportion of patients with fever associated with signs suggestive of other diseases, but also suffering from malaria, was unknown.

Attempts to quantify the malaria-attributable fraction, the proportion of fevers among people with malaria infection that are actually due to malaria, have been made by a number of authors, using both cross-sectional household surveys and health facility-based patient enrolment, both to determine malaria-attributable fraction for a population through modelling and to determine age and seasonbased cut-offs, though this was found to vary greatly by age, season and transmission intensity [5-9]. Malaria case management has historically been based on clinical diagnosis in resource-poor settings, resulting in massive overdiagnosis and overtreatment of malaria [10-13]. Some have attempted to develop rule-based systems to diagnose malaria clinically, but the sensitivity and specificity remain poor [14-16]. There has been no other documented experience of a country adopting a case management algorithm that included clinical assessment along with fever or history of fever for recommendation of malaria testing.

This study was designed to evaluate the first step of the case management algorithm, which recommends testing for malaria if no signs or symptoms of an alternative fever source are present, as a screening test, considering the RDT as the definitive test, to determine the sensitivity, specificity, positive predictive value, and negative predictive value of limiting malaria RDT testing based on the first step of this algorithm, compared to universal RDT testing for febrile patients throughout the malaria transmission zones of Senegal and throughout the year to guide case management policy. These values were to be determined for patients under 5 years old and 5 years and above, during rainy and dry season, and in each of four malaria transmission zones.

\section{Methods \\ Study design}

A longitudinal health facility-based study was conducted at 16 health posts in eight health districts, with two health districts in each of the four malaria transmission zones considered by the NMCP: north (low transmission, reported annual malaria incidence $<5 / 1000$ inhabitants); centre (moderate transmission, reported annual malaria incidence 5-35/1000 inhabitants); south (high transmission, reported annual incidence $>35 / 1000$ inhabitants); and, the capital city of Dakar (heterogeneous) (Fig. 2). Health posts were chosen by district health officers to have median use and to be typical of the district. 


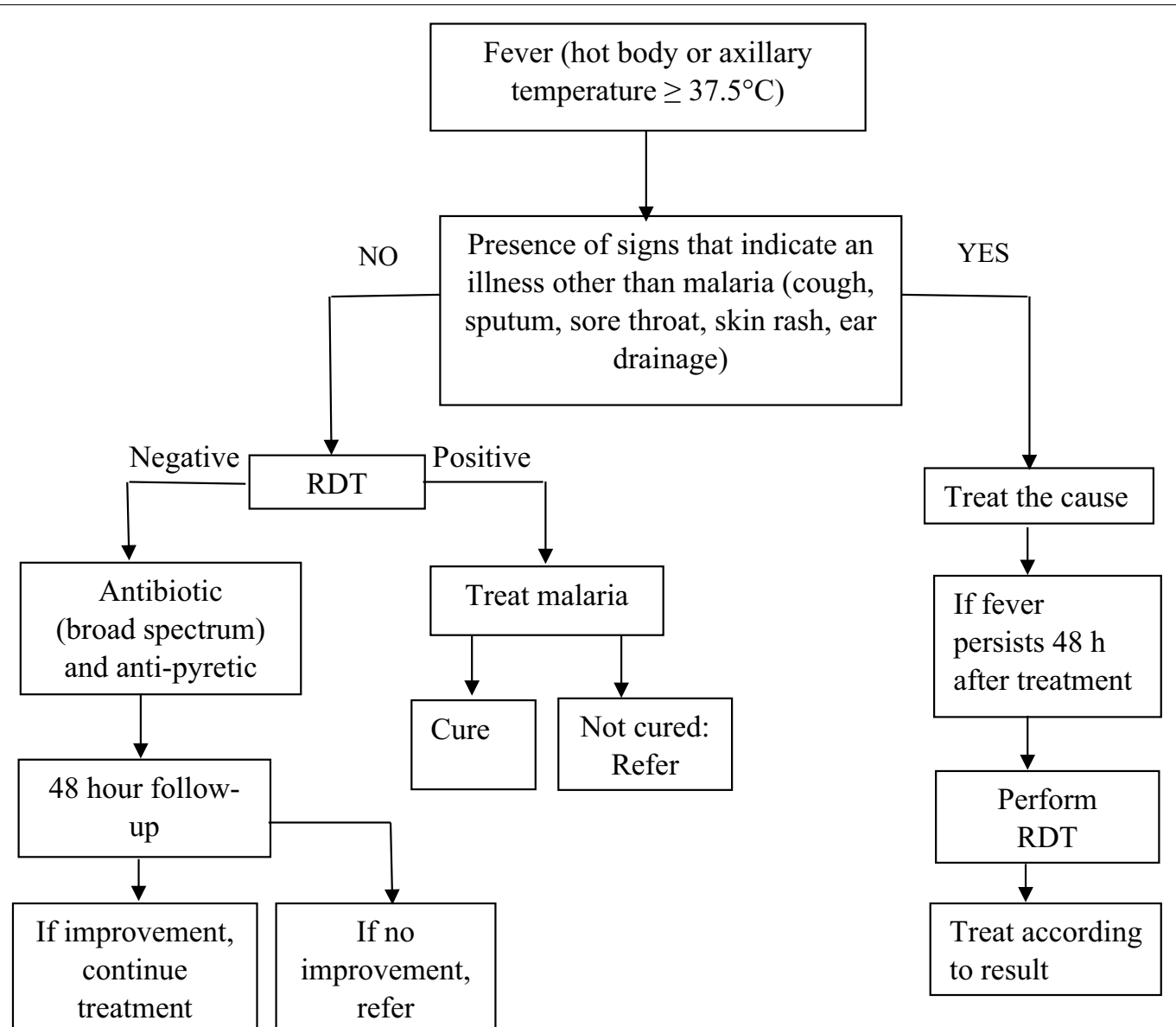

Fig. 1 Senegal National Malaria Control Program case management algorithm for patients of all ages with uncomplicated febrile illness or history of fever in Senegal

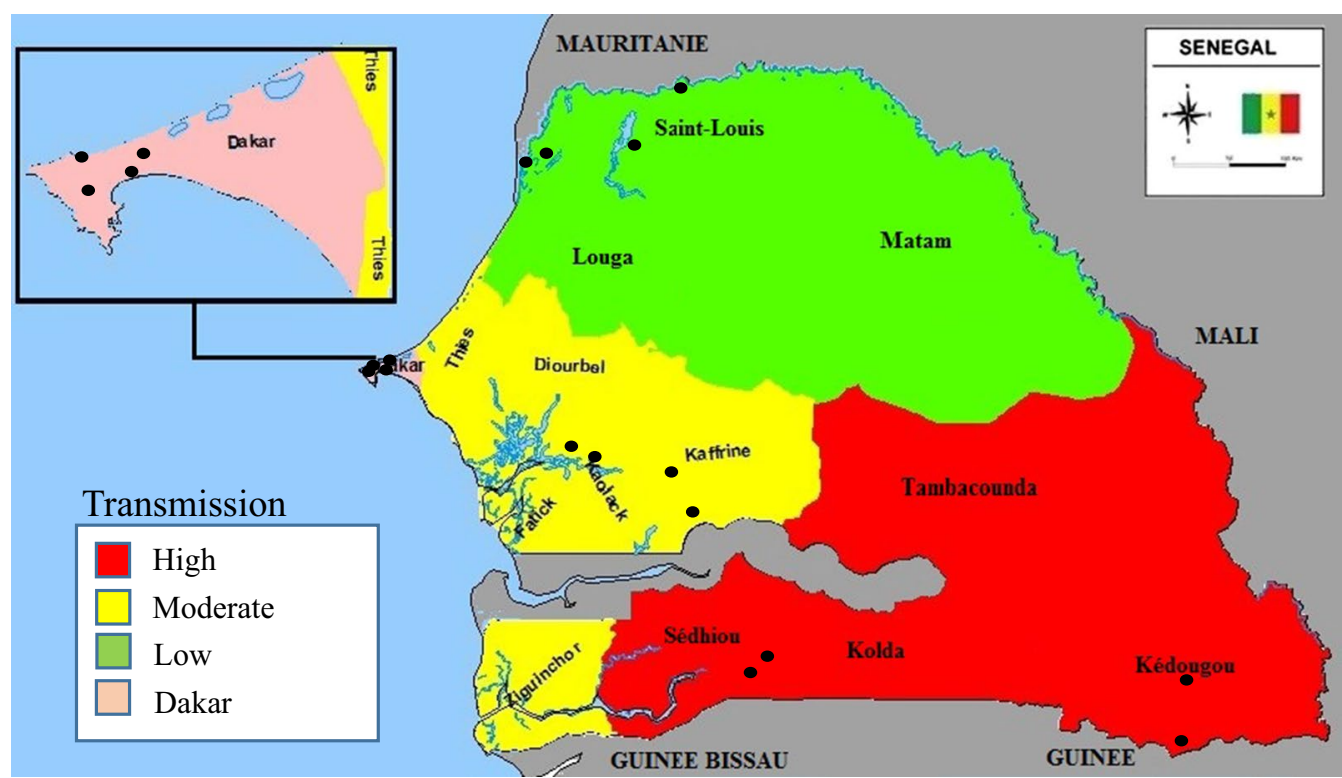

Fig. 2 Map of study sites by transmission zone. Filled circle study site. Source: Senegal National Malaria Control Program 
In order to have an adequate sample size to analyse results by age group ( $<5$ and $\geq 5$ years), season (rainy and dry), and transmission zone (high, moderate, low, heterogeneous/Dakar), with a 95\% confidence interval, with a margin of $5 \%$, at least 369 patients were required to be enrolled at each site. The sample size was set at 6144 , with each of the 16 health posts expected to enrol 32 patients monthly for 12 months ( 384 per site).

\section{Enrolment}

Health post staff were trained on study methods and conducted the enrolment. During a full year (April 2013-March 2014), health post staff recruited patients 4 days per week, enrolling the first patient of age at least 2 months (infants younger than 2 months are excluded from the algorithm) but younger than 5 years and the first patient 5 years or older each day of enrolment who presented with fever (axillary temperature of $\geq 37.5{ }^{\circ} \mathrm{C}$ ) or history of fever in the previous $48 \mathrm{~h}$, for their first visit to the health facility for that illness. If a febrile patient of either age group did not present on a given day of enrolment, catch-up was allowed on other days of the week. Pregnant women and patients with signs of severe disease were excluded, as they are excluded from the algorithm.

All recruited patients gave informed consent, or consent of caregiver in the case of minors. Health post staff then completed a questionnaire documenting region, district, health facility, age, gender, duration of symptoms, previous care sought, temperature, symptoms, and physical examination. Based on these, they determined whether testing with an RDT would be recommended by the algorithm, and documented their determination. Following this, all enrolled patients were tested with RDT (SD BIOLINE Malaria Ag Pf HRP-2), with results and treatment documented. During the first 9 months of the study, as an RDT quality control measure for patients enrolled on 2 of the 4 days each week, a thick blood film was also performed for quality control. All patients were treated according to the result of the RDT with an ACT for positive tests as directed by national policy, with ancillary therapy as indicated. All patients were given an appointment for follow-up in $48 \mathrm{~h}$.

\section{Laboratory procedures}

Every month, a team composed of a research assistant and a laboratory technician visited every health post, reviewed study materials, collected questionnaires and blood films, did any needed trouble shooting, and supervised and corrected blood film technique. Blood films were dried and labelled with pre-printed ID stickers on site, and were stained in 10\% Giemsa for $15 \mathrm{~min}$ and read in Dakar by expert malaria microscopists in the national reference laboratory (University Cheikh Anta Diop
Parasitology Department). Two parasitologists read the films in a blinded manner, with a third reading in case of discrepancy between the results of the two readings. In case of discrepancy, the third microscopist's reading was used. There were discrepant readings on five slides. Asexual malaria parasites were enumerated against 200-500 white blood cells (WBC), transformed to parasites $/ \mu \mathrm{L}$ using the assumption of $8000 \mathrm{WBC} / \mu \mathrm{L} ; 250$ fields were read before declaring any film negative.

\section{Data management}

Questionnaires were entered into an Epi-Info (version 7.1.4, CDC, Atlanta, GA, USA) database by double entry. Every quarter, the research team met to review the data and address any data quality issues. Data were analysed using Epi Info.

The sample was restricted to patients enrolled from 1 April, 2013 to 31 March, 2014. During some months, health post personnel, trying to make up for months during which they had not enrolled the desired sample size per month, enrolled a large number of patients. Up to double the month's quota of 32 were accepted. For any month during which a site enrolled more than 64 patients, enrollees were randomly selected from the highest enrolment days for removal. This exercise was performed for three health posts: two during July and one during August.

\section{Analysis}

Rainy season was defined as July-December and dry season was defined as January-June. Fever was defined as an axillary temperature of $\geq 37.5{ }^{\circ} \mathrm{C}$, and history of fever was defined as a complaint of a hot body in the previous $48 \mathrm{~h}$. In order to determine if health providers had implemented the algorithm and correctly assigned patients to the groups to be tested, two senior members of the research team reviewed the symptoms recorded for each patient and determined whether the algorithm recommended testing. Concordance between health post workers and expert review was assessed. Sensitivity, specificity, positive predictive value, and negative predictive value of the algorithm as a screening test were calculated, compared to RDT. This calculation was done across age groups, transmission zones and rainy/dry season to determine for which age groups, in which transmission zones, and for which seasons the algorithm demonstrated sufficient sensitivity to diagnose an adequate proportion of febrile patients with parasitaemia.

\section{Ethical aspects}

The protocol was reviewed and approved by the Senegal National Ethical Committee (Comité National d'Ethique pour la Recherche Santé \#0109) and by the Centers for 
Disease Control and Prevention Human Subjects Board, where it received a non-research determination. Participation was strictly voluntary. Patients who refused consent received the same evaluation and treatment as they would have if they had participated, other than an RDT for patients that would not have met criteria for RDT according to the algorithm.

\section{Results}

\section{Enrolment and demographics}

From April 2013 to March 2014, 6039 patients were enrolled. Of these, $46.5 \%$ (95\% CI 45.2-47.7) were children under five, reflective of the instructions given, and $48.4 \%$ were male (95\% CI 47.2-49.7). Of the 5988 for whom data were recorded, $32.2 \%$ (95\% CI 31.0-33.4) sought initial care elsewhere prior to this consultation. Of 6035 for whom temperature was recorded, 83.1\% (95\% CI 82.1-84.0) were febrile at consultation, with a median duration of symptoms of 2.4 days at the time of consultation. The breakdown by quarter and malaria risk zone is found in Table 1. While approximately equal numbers of patients were enrolled in each risk zone, $44.1 \%$ of patients were enrolled during the dry season (January-June), compared to $55.9 \%$ during the rainy season (July-December).

\section{Need for testing as determined by algorithm}

Overall, 57.7\% (95\% CI 56.4-58.9) (3483/6039) were determined eligible to receive an RDT according to the algorithm (Fig. 1), which varied by age group, season and malaria transmission risk zone (Table 2). Of children under 5 years, $44.4 \%$ (95\% CI 42.6-46.3) were determined eligible to be tested, compared to $69.2 \%$ (95\% CI 67.6-70.8) of patients 5 years or older. The percent of patients eligible to be tested by malaria risk zone ranged from $50.8 \%$ (heterogeneous) to $64.5 \%$ (high). With the exception of the high transmission risk zone, in which $54.2 \%$ (95\% CI 44.8-52.4) were to be tested in dry season compared to $72.0 \%$ (95\% CI 68.8-74.9) in rainy season, the difference from rainy to dry season was minimal. Concordance between the health post workers and study team regarding patients requiring testing according to the algorithm was $90.0 \%$ (95\% CI 89.2-90.7).

\section{Rapid diagnostic test positivity}

Regardless of whether they were eligible for an RDT or not according to the algorithm, all enrolled patients were tested for malaria with an RDT; results were available for 6038/6039. Overall, 22.7\% (95\% CI 21.7-23.8) were positive for malaria. RDT positivity rate was $11.7 \%$ (95\% CI $10.5-$ 13.0) (298/2554) for those for whom an RDT was not recommended by the first step of the algorithm, compared to $30.9 \%$ (95\% CI 29.4-32.4) (1075/3483) for those for whom an RDT was recommended by the algorithm. Children under 5 years had an RDT positivity rate of $16.5 \%(95 \%$ CI 15.1-17.9) compared to $28.2 \%$ (95\% CI 26.7-29.8) for patients 5 years or older. The positivity rate noted by risk zone confirmed the highly stratified epidemiology. In the low risk northern zone, the RDT positivity rate was only $1.3 \%$ (95\% CI $0.8-2.1$ ), varying from $0.5 \%$ (95\% CI $0.1-1.5$ ) in the dry season to $2.0 \%$ (95\% CI 1.2-3.2.) in the rainy season. In the high-risk southern zone, the RDT positivity rate was $56.5 \%$ (95\% CI 53.9-59.0), varying from $27.1 \%$ (95\% CI $23.7-30.9)$ in the dry season to $77.6 \%$ (95\% CI 74.7-80.3) in the rainy season. The centre (moderate) and Dakar (heterogeneous) were intermediate in RDT positivity, with a fivefold difference from dry to rainy season (Table 3).

\section{Microscopy for RDT quality assurance}

During the first 9 months of the study (April-December 2013), blood films were collected for $49.3 \%$ of patients (2396/4856), and 23.3\% (21.7-25.1) of blood films were positive, compared to $25.5 \%$ (95\% CI $24.3-26.7$ ) of RDTs from this period. All positive samples were identified as Plasmodium falciparum; no other malaria parasite species were detected by microscopy. Sensitivity and specificity of RDTs, compared to blood films (of the 2396 RDTs performed during the first 9 months), were both 99.1\%, demonstrating excellent reliability in the hands of well-trained health post staff.

\section{Characteristics of the diagnostic algorithm}

Overall, sensitivity of the first step of the diagnostic algorithm (based on facility health workers' assessments) compared to RDT was 78.3\% (95\% CI 76.0-80.4), specificity was $48.4 \%$ (95\% CI $46.9-49.8$ ), negative predictive value was $88.3 \%$ (95\% CI $87.2-89.4$ ), and positive predictive

Table 1 Distribution of patients enrolled by trimester and malaria transmission risk zone

\begin{tabular}{|c|c|c|c|c|c|}
\hline & Jan-Mar & Apr-Jun & Jul-Aug & Oct-Dec & Total \\
\hline North (low) & 248 & 371 & 443 & 371 & $1433(24 \%)$ \\
\hline Centre (moderate) & 350 & 394 & 418 & 376 & $1538(25 \%)$ \\
\hline South (high) & 261 & 366 & 533 & 334 & $1494(25 \%)$ \\
\hline Dakar (heterogeneous) & 320 & 353 & 522 & 378 & $1573(26 \%)$ \\
\hline Total & 1179 (20\%) & $1484(25 \%)$ & $1916(32 \%)$ & $1459(24 \%)$ & 6038 \\
\hline
\end{tabular}




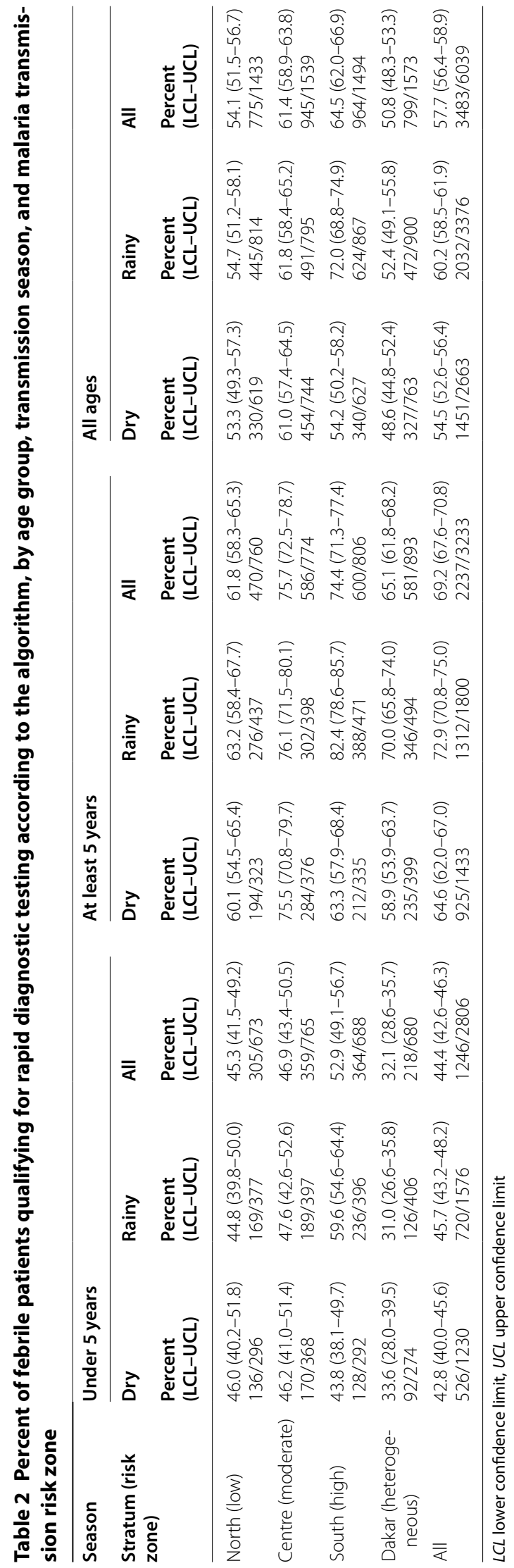




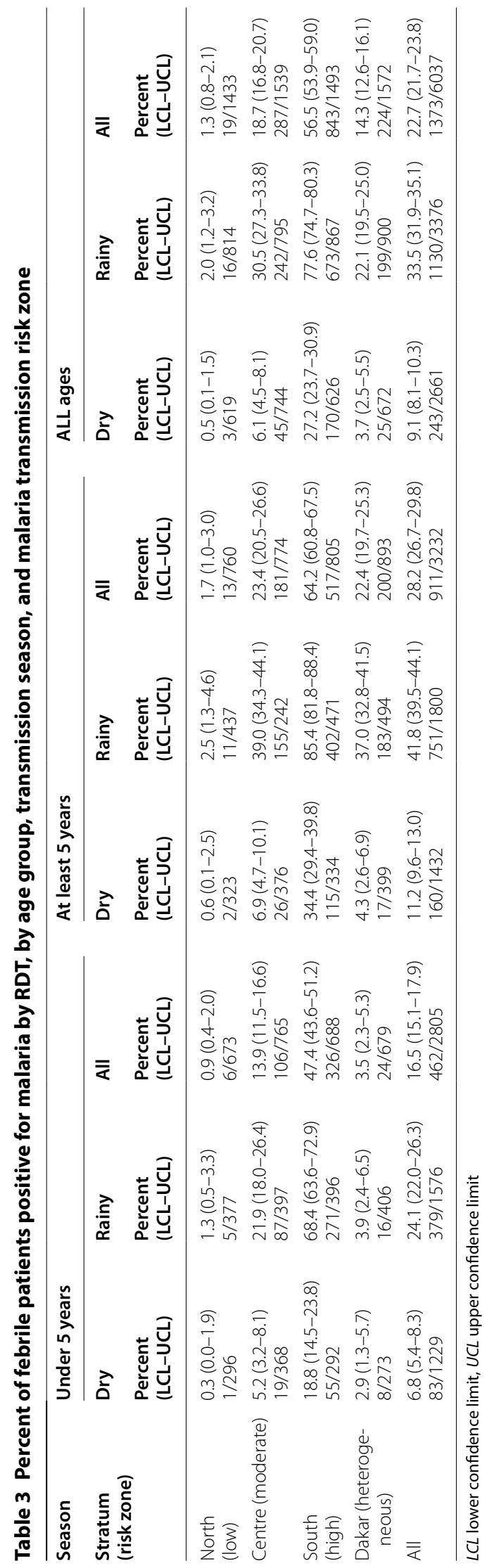


value was $30.9 \%$ (95\% CI 30.0-31.7). Calculation of the diagnostic values for both age groups, rainy and dry season, and every transmission risk zone separately demonstrated large differences in diagnostic values depending on these categories (Table 4). The sensitivity of the algorithm followed transmission intensity, with the greatest sensitivity (but wide confidence intervals, due to low numbers of malaria cases) in areas of lowest transmission (north), progressively decreasing with increasing transmission, and the lowest sensitivity (and narrower confidence intervals) in the south. Sensitivity in the north, calculated for the entire year, was $83.3 \%$ (95\% CI 36.9-99.6) among children under 5 years and $100.0 \%$ (95\% CI 75.3-100.0) among patients 5 years and older. By comparison, in the south, sensitivity calculated for the entire year was $64.7 \%$ (95\% CI 59.3-70.0) among children under 5 years, and 81.0\% (95\% CI 77.484.3) among patients 5 years and older. The moderate transmission centre fell between these, with sensitivity calculated for year of $75.5 \%$ (95\% CI 66.2-83.3) among children under 5 years and 85.1\% (95\% CI 76.6-85.9) among patients 5 years and older. In every season and risk zone, sensitivity among children under 5 years was less than that for patients 5 years and older, with sensitivity calculated all year and nationwide of $67.5 \%$ (95\% CI 63.1-71.8) among children under 5 years and $83.8 \%$ (95\% CI 81.2-86.1) among patients 5 years and older. No clear seasonal trend regarding sensitivity emerged. Outside the north, sensitivity did not surpass $90 \%$ in any age group, season or risk zone. While specificity was low throughout, as the first step of the algorithm is used as a screening test, a certain level of false positivity is desirable.

Both positive and negative predictive values were highly associated with season and malaria transmission risk zone. Negative predictive value was high during the dry season and in lower transmission zones, and was consistently higher among children under 5 than among patients 5 years and older. With the exception of the high transmission south, it was consistently greater than $95 \%$ during dry season and above $75 \%$ in rainy season. In the south during the rainy season, negative predictive value plunged to $24.8 \%$ (95\% CI 17.1-32.9) among patients 5 years and older, and $45.0 \%$ (95\% CI 39.4-50.7) among children under 5 years. Given that malaria incidence is generally low in Senegal, positive predictive value was relatively low, save in the south during rainy season, where it was $77.5 \%$ (95\% CI 73.5-81.2) among children under 5 years and $87.4 \%$ (85.5-89.0) among patients 5 years and older.

The sensitivity of the study team's determination of RDT eligibility according to the algorithm compared to RDT was determined to be $82.8 \%$ (95\% CI 80.7-84.8), compared to $78.3 \%$ (95\% CI 76.0-80.4) for the determination by the health post workers. Specificity was $39.4 \%$
(95\% CI $38.0-40.8$ ), compared to $48.4 \%$ (95\% CI $46.9-$ 49.8) for the health post workers. Other diagnostic values were within $5 \%$ points.

Considering only patients for whom a blood film was positive ( $\mathrm{n}=621$ ), 22\% (137) had been judged not to be eligible to receive an RDT. Patients for whom a blood film was positive but who were judged not eligible to receive an RDT had a mean parasite density of 23,075 parasites/ $\mu \mathrm{L}$ (range 480-288,000), while patients with positive smear who had been judged eligible to receive an RDT had a mean parasite density of 22,639 parasites/ $\mu \mathrm{L}$ (range $400-408,000)$. For all positive smears, mean and median parasite density were similar for children under 5 years (mean 22,176; median 5000), children 5-9 years (mean 26,324; median 5078), and patients 10 years and older (mean 21,636; median 6045).

\section{Follow-up}

All patients were given a 48-h follow-up appointment, which $70.0 \%$ kept. Only eight $(0.2 \%)$ were judged to have had a non-favourable evolution, two of whom had had a positive RDT and had been treated with ACT. Of the five patients that had a negative RDT but positive blood film on the initial visit, parasite density was available for four and ranged from 667 to 10,160 parasites $/ \mu \mathrm{L}$ at the initial visit. Four of the five attended the 48-h follow-up visit, and were all judged to have had a favourable clinical evolution.

\section{Discussion}

Senegal was one of the first countries in sub-Saharan Africa to recommend laboratory-confirmed malaria diagnosis at all health posts nationwide and was forced to do so in the context of resource constraints. The case management algorithm, as it was designed, represented the consensus of malaria scientists and programme managers in Senegal as the best way to use limited resources to diagnose the greatest number of malaria sufferers. It was implemented with the intention to evaluate and modify it as the situation evolved.

This study was the first to measure year-round malaria test positivity rate among patients with uncomplicated febrile illness throughout Senegal, and confirms what has been seen in the routine health information system and cross-sectional surveys regarding the highly seasonal and graduated nature of malaria transmission in Senegal. The RDT positivity rate was very low during dry season and in the north, and quite high in the south in rainy season. While the proportion of patients eligible to be tested according to the algorithm (patients without another fever source) varied by transmission risk zone, the variation was within $15 \%$ points from north to south, and the variation between dry and rainy transmission seasons 
Table 4 Diagnostic values of case management algorithm, by age group, transmission season, and malaria transmission risk zone

\begin{tabular}{|c|c|c|c|c|c|c|c|c|c|c|}
\hline Risk zone & $\begin{array}{l}\text { Age group } \\
\text { (years) }\end{array}$ & Season & $\mathrm{TP}$ & FP & FN & TN & $\begin{array}{l}\text { SENS } \\
\%(95 \% \mathrm{Cl})\end{array}$ & $\begin{array}{l}\text { SPEC } \\
\%(95 \% \mathrm{CI})\end{array}$ & $\begin{array}{l}\text { NPV } \\
\%(95 \% \mathrm{Cl})\end{array}$ & $\begin{array}{l}\text { PPV } \\
\%(95 \% \mathrm{Cl})\end{array}$ \\
\hline \multirow[t]{6}{*}{ North (low) } & \multirow[t]{3}{*}{$<5$} & Dry & 1 & 135 & 0 & 160 & $100.0(2.5-100.0)$ & $54.2(48.4-60.0)$ & $100.0\left(^{\mathrm{a}}\right)$ & $0.7(0.7-0.8)$ \\
\hline & & Rainy & 4 & 165 & 1 & 207 & $80.0(28.4-99.5)$ & $55.6(50.4-60.8)$ & 99.5 (97.3-99.9) & $2.4(1.5-3.7)$ \\
\hline & & All year & 5 & 300 & 1 & 367 & 83.3 (36.9-99.6) & $55.0(51.2-58.8)$ & $99.7(98.4-100.00)$ & $1.6(1.1-2.4)$ \\
\hline & \multirow[t]{3}{*}{$\geq 5$} & Dry & 2 & 192 & 0 & 129 & $100.0(15.8-100.0)$ & $40.2(34.8-45.8)$ & $100.0\left(^{a}\right)$ & $1.0(0.9-1.1)$ \\
\hline & & Rainy & 11 & 265 & 0 & 161 & $100.0(71.5-100.0)$ & $37.8(33.2-42.6)$ & $100.0\left({ }^{a}\right)$ & $4.0(3.7-4.3)$ \\
\hline & & All year & 13 & 457 & 0 & 290 & $100.0(75.3-100.0)$ & $38.8(35.3-42.4)$ & $100.0\left({ }^{\mathrm{a}}\right)$ & $2.8(2.6-2.9)$ \\
\hline \multirow[t]{6}{*}{ Centre (mod) } & \multirow[t]{3}{*}{$<5$} & Dry & 14 & 156 & 5 & 193 & $73.7(48.8-90.9)$ & $55.3(49.9-60.6)$ & $97.5(94.5-98.8)$ & $8.2(6.3-10.7)$ \\
\hline & & Rainy & 66 & 123 & 21 & 187 & $75.9(65.5-84.4)$ & $60.3(54.6-65.8)$ & $89.9(85.9-92.9)$ & $34.9(30.9-39.2)$ \\
\hline & & All year & 80 & 279 & 26 & 380 & 75.5 (66.2-83.3) & $57.7(53.8-61.5)$ & $93.6(91.2-95.4)$ & $22.3(20.0-24.8)$ \\
\hline & \multirow[t]{3}{*}{$\geq 5$} & Dry & 22 & 262 & 4 & 88 & 84.6 (65.1-95.6) & $25.1(20.7-30.0)$ & $95.7(89.8-98.2)$ & $7.7(6.6-9.1)$ \\
\hline & & Rainy & 132 & 170 & 23 & 72 & $85.2(78.6-90.4)$ & $29.8(24.1-35.9)$ & $75.8(67.2-82.7)$ & $43.7(41.1-46.3)$ \\
\hline & & All year & 154 & 432 & 27 & 160 & 85.1 (79.0-89.9) & $27.0(23.5-30.8)$ & $85.6(80.3-89.6)$ & $26.3(24.8-27.8)$ \\
\hline \multirow[t]{6}{*}{ South (high) } & \multirow[t]{3}{*}{$<5$} & Dry & 28 & 100 & 27 & 137 & $50.9(37.1-74.7)$ & $57.8(51.2-64.2)$ & $83.5(79.2-87.2)$ & $21.9(17.2-27.4)$ \\
\hline & & Rainy & 183 & 53 & 88 & 72 & $67.5(61.6-73.1)$ & $57.6(48.4-66.4)$ & $45.0(39.4-50.7)$ & $77.5(73.5-81.2)$ \\
\hline & & All year & 211 & 153 & 115 & 209 & $64.7(59.3-70.0)$ & $57.7(52.5-62.9)$ & $64.5(60.5-68.3)$ & $58.0(54.4-61.4)$ \\
\hline & \multirow[t]{3}{*}{$\geq 5$} & Dry & 80 & 132 & 35 & 87 & $69.6(60.3-77.8)$ & $39.7(33.2-46.5)$ & $71.3(64.3-77.4)$ & $37.7(34.0-41.6)$ \\
\hline & & Rainy & 339 & 49 & 63 & 20 & $84.3(80.4-87.7)$ & $29.0(18.7-41.2)$ & $24.1(17.1-32.9)$ & $87.4(85.5-89.0)$ \\
\hline & & All year & 419 & 181 & 98 & 107 & $81.0(77.4-84.3)$ & $37.2(31.6-43.0)$ & $52.2(46.4-58.0)$ & $69.8(67.7-71.9)$ \\
\hline \multirow[t]{6}{*}{ Dakar (mixed) } & \multirow[t]{3}{*}{$<5$} & Dry & 7 & 85 & 1 & 180 & 87.5 (47.4-99.7) & $67.9(61.9-73.5)$ & $99.4(96.6-99.9)$ & $7.6(5.7-10.1)$ \\
\hline & & Rainy & 9 & 117 & 7 & 273 & $56.3(29.9-80.3)$ & $70.0(65.2-80.3)$ & 97.5 (95.7-98.6) & $7.1(4.6-10.8)$ \\
\hline & & All year & 16 & 202 & 8 & 453 & $66.7(44.7-84.4)$ & $69.2(65.5-72.7)$ & $98.3(97.0-99.0)$ & $7.3(5.5-9.7)$ \\
\hline & \multirow[t]{3}{*}{$\geq 5$} & Dry & 15 & 220 & 2 & 162 & $88.2(63.6-98.5)$ & $42.4(37.4-47.5)$ & $98.8(95.6-99.7)$ & $6.4(5.3-7.6)$ \\
\hline & & Rainy & 162 & 184 & 21 & 127 & 88.5 (83.0-92.8) & $40.8(35.3-46.5)$ & $85.8(79.8-90.2)$ & $46.8(44.2-49.5)$ \\
\hline & & All year & 177 & 404 & 23 & 289 & 88.5 (83.3-92.6) & $41.7(38.0-45.4)$ & 92.6 (89.4-94.9) & $30.5(28.8-32.2)$ \\
\hline
\end{tabular}

$\mathrm{TP}$, true positive: algorithm recommends testing, and RDT is positive; $\mathrm{FP}$, false positive: algorithm recommends testing, and RDT is negative; $\mathrm{FN}$, false negative: algorithm does not recommend testing, and RDT and positive; TN, true negative: algorithm does not recommend testing, and RDT is negative

SENS sensitivity, SPEC specificity, NPV negative predictive value, PPV positive predictive value

a Unable to calculate

was only $5 \%$ points, with roughly $60 \%$ of febrile patients recommended for testing according to the algorithm.

Surprisingly, children under 5 years old were substantially less likely than patients 5 years and older to be determined eligible for a test, though young children have frequent fevers due to many upper respiratory and other infections, which diminish with age, while adults have fever more rarely, and those fevers may be more likely to be considered malarial.

While this study was not an attempt to estimate malaria-attributable fraction, the diagnostic algorithm may be considered a method to help healthcare providers identify patients among whom fever is likely to be attributable to malaria. The RDT positivity rate was indeed higher, by almost three-fold, among those recommended for RDT testing than among those not recommended for RDT testing by the first step of the algorithm. Studies that examined the effect of seasonality found a higher malaria-attributable fraction during the rainy season $[7,9]$ and studies that looked at the effect of transmission intensity found that a higher proportion of fevers in higher transmission zones were due to malaria $[5,6,8]$, as was found in Senegal. These assumptions were generally confirmed by the proportion of positive tests by season and transmission zone. However, the findings both that febrile children under 5 years were judged by healthcare providers to be more likely to have a fever source other than malaria and that children under 5 years actually did have a lower test positivity rate stand in contrast to other studies, regardless of transmission zone. In neighbouring Mali and Burkina Faso, as well as in Kenya and Mozambique, younger children had a higher malaria-attributable fraction for fever and higher test positivity rates than older children and adults [5, 7, 9, 17]. Moreover, febrile children under 5 years had a lower test positivity rate 
than febrile patients 5 years and older during both rainy and dry seasons in every transmission zone in this study.

While sensitivity and specificity for laboratory tests are theoretically intrinsic and unchanging values, for a case management algorithm there is variation; this algorithm is a way of assigning testing to those with higher pre-test probability. The sensitivity of the algorithm for identifying patients with malaria among patients under 5 years was lower than that for patients 5 years or older, despite the lower test positivity rate among children under 5 years, and was lowest in the highest transmission risk zone. While the diagnostic algorithm permits testing and diagnosis of almost $80 \%$ of febrile patients with malaria parasites nationwide over the course of the year, this proportion is unacceptably low for use as a screening test. In addition, in high transmission zones during the dry season, just over one half of children under 5 years with parasites would be detected by the diagnostic algorithm, validating the complaints of the health providers that if they follow the algorithm, they miss too many cases of malaria. Low specificity, on the other hand, would not be as problematic for a screening test. Negative predictive value is generally greater than $85 \%$, consistent with relatively low burden, except for in the high transmission zone during the rainy season, where it falls to only 45 and $24 \%$ among children under 5 years and among patients 5 years and older, respectively; while otherwise low, positive predictive value is greater than $75 \%$ among all ages in the high transmission zone during rainy season.

Some of the patients ineligible for a test according to the diagnostic algorithm that actually had malaria parasites may in fact have had symptoms due to another cause, with simultaneous asymptomatic malaria parasitaemia. When the algorithm was adopted, it was assumed that these patients likely had low density parasitaemia not requiring treatment. However, among patients for whom a blood film was obtained, and among whom that film was positive, patients for whom an RDT was recommended and patients for whom an RDT was not recommended had very similar mean parasite densities. As Senegal moves toward the goal of pre-elimination, every opportunity to diagnose and treat patients with parasites becomes increasingly important. Senegal is adopting strategies of reactive active case detection, even testing asymptomatic household contacts in elimination zones [18]. Theoretically, as exposure to parasites decreases and immunity wanes, the likelihood of truly asymptomatic parasitaemia decreases, although this decrease has not been found to be as profound as expected; evidence suggests that $60 \%$ of infections, even at low transmission, are asymptomatic [19].

This study had several limitations. Due to budgetary constraints, health post nurses were trained to carry out the study procedures instead of hiring dedicated study staff. Hence, a simplified enrolment scheme was used, and enrolment was dependent on the care providers' comprehension of procedures as well as work schedule, though it enabled understanding of how the care providers were evaluating patients. Providers struggled to enrol the planned numbers of patients during the dry season, and among children under 5 years, and were not required to report total numbers of patients or total numbers of febrile patients seen on days of enrolment. Results were presented by age group, season and transmission stratum separately to minimize bias due to this. The limitation to 16 health posts made it impossible to get a nationally representative sample, however sites from across all four of the malaria transmission risk zones were included. While relying on a care provider to assess the need for an RDT according to the algorithm may have been a weakness, the classification by study personnel, including a member of the NMCP, had good concordance with that of the provider. Concern for another potential weakness, that of using RDT for gold standard diagnosis, was mitigated by the very high sensitivity and specificity of RDT compared to reference laboratory microscopy for the half of the samples for which a blood slide was obtained.

\section{Conclusions}

Despite the limitations, this study provided unequivocal evidence that the first step of the case management algorithm caused providers who followed it strictly to miss an unacceptable proportion of malaria infections. Based on the poor sensitivity of the diagnostic algorithm, the Senegal NMCP is phasing out the use of the first step of the algorithm in favour of universal testing of febrile patients, while rolling out the policy over several years to assure that adequate RDT supplies could be ordered to support the new policy. Starting in 2015, all febrile children under 5 years nationwide and year-round were to be tested with an RDT, while universal testing for febrile patients 5 years or older was limited to the rainy season. The routine malaria information system in Senegal demonstrated that the number of children under 5 years tested for malaria increased from 136,847 in 2014 to 364,771 in 2015 [20, 21]. Starting in 2017, febrile patients of all ages will be tested for malaria throughout the year.

\footnotetext{
Authors' contributions

$J T, M B D, F B, A B G, S G, M N, M C$, and $M B$ participated in study design, and oversaw implementation, interpretation and dissemination of results. $A D$, AS and GS were responsible for field implementation of the study and presentation of the results to in-country stakeholders. AD and YD visited health posts monthly for supervision. YD and DN assured RDT and blood slide quality and oversaw all laboratory aspects. JT and FB made the determination as to whether the patients required an RDT according to the algorithm. JT performed analysis and interpretation of results, and drafted the manuscript, which was reviewed by all co-authors. All authors read and approved the final manuscript.
} 


\begin{abstract}
Author details
${ }^{1}$ U.S. Centers for Disease Control and Prevention and President's Malaria Initiative, Atlanta, USA. ${ }^{2}$ Senegal National Malaria Control Programme, Dakar Senegal. ${ }^{3}$ Pediatrics Service Hôpital le Dantec, Dakar, Senegal. ${ }^{4}$ Parasitology Service Hôpital le Dantec, Dakar, Senegal. ${ }^{5}$ United States Agency for International Development, Dakar, Senegal. ${ }^{6}$ Université Cheikh Anta Diop, Dakar, Senegal. ${ }^{7} \mathrm{WHO}$, Dakar, Senegal.
\end{abstract}

\section{Acknowledgements}

We would like to thank all the health post nurses, who added these responsibilities to their already full workload, and all the patients and their caregivers who consented to participate.

\section{Competing interests}

The authors declare that they have no competing interests.

\section{Availability of data and materials}

The datasets used and/or analysed during the current study are available from the corresponding author on reasonable request.

\section{Ethics approval and consent to participate}

The protocol was reviewed and approved by the Senegal National Ethical Committee (Comite National d'Ethique pour la Recherche Sante \#0109) and by the Centers for Disease Control and Prevention Human Subjects Board, where it received non-research determination. All participants or their caregivers gave written informed consent.

\section{Funding}

This work was made possible through support provided by the President's Malaria Initiative (PMI), and the US Agency for International Development, under the terms of an Interagency Agreement with the Centers for Disease Control and Prevention (CDC). A representative of PMI participated in design, implementation, analysis, and interpretation of results. The opinions expressed herein are those of the authors and do not necessarily reflect the views of the US Agency for International Development or CDC

\section{Received: 3 November 2016 Accepted: 24 February 2017} Published online: 01 March 2017

\section{References}

1. Ministère de la Santé, de la Prévention et de l'Hygiène Publique/PNLP. Capitalisation de l'introduction des ACT au Sénégal. Rapport Etude IPDSR/UCAD. 2009

2. Ministère de la Santé et de la Prévention Médicale/PNLP. Etude de la faisabilité des TDR au Sénégal. Rapport Etude du Laboratoire de Parasitologie de I'UCAD. 2007

3. Ministère de la Santé, de la Prévention et de l'Hygiène Publique/PNLP. Capitalisation de l'introduction des TDR au Sénégal. Rapport Etude IPDSR/UCAD. 2009

4. Thiam S, Thior M, Faye B, Ndiop M, Diouf ML, Diouf MB, et al. Major reduction in anti-malarial drug consumption in Senegal after nation-wide introduction of malaria rapid diagnostic tests. PLOS ONE. 2011;6:e18419.

5. Afrane YA, Zhou G, Githeko AK, Yan G. Clinical malaria case definition and malaria attributable fraction in the highlands of western Kenya. Malar J. 2014;13:405.
6. Tchuinkam T, Nyih-Kong B, Fopa F, Simard F, Antonio-Nkondjio C, AwonoAmbene HP, et al. Distribution of Plasmodium falciparum gametocytes and malaria-attributable fraction of fever episodes along an altitudinal transect in Western Cameroon. Malar J. 2015;14:96

7. Dicko A, Mantel C, Kouriba B, Sagara I, Thera MA, Doumbia S, et al. Season, fever prevalence and pyrogenic threshold for malaria disease definition in an endemic area of Mali. Trop Med Int Health. 2005;10:550-6.

8. Mabunda S, Aponte JJ, Tiago A, Alonso P. A country-wide malaria survey in Mozambique. II. Malaria attributable proportion of fever and establishment of malaria case definition in children across different epidemiological settings. Malar J. 2009;8:74

9. Bisoffi Z, Sirima SB, Menten J, Pattaro C, Angheben A, Gobbi F, et al. Accuracy of a rapid diagnostic test on the diagnosis of malaria infection and of malaria-attributable fever during low and high transmission season in Burkina Faso. Malar J. 2010;9:192.

10. WHO. Guidelines for the treatment of malaria. Geneva: World Health Organization; 2006. http://www.afro.who.int/en/downloads/cat_ view/1501-english/1235-clusters-and-programmes/795-malaria-mal. html?start=15. Accessed 23 Sep 2016

11. D'Acremont V, Kahama-Maro J, Swai N, Mtasiwa D, Genton B, Lengeler C. Reduction of anti-malarial consumption after rapid diagnostic tests implementation in Dar es Salaam: a before-after and cluster randomized controlled study. Malar J. 2011;10:107

12. Rao VB, Schellenberg D, Ghani AC. Overcoming health systems barriers to successful malaria treatment. Trends Parasitol. 2013;29:164-80.

13. Orish VN, Ansong JY, Onyeabor OS, Sanyaolu AO, Oyibo WA, Iriemenam NC. Overdiagnosis and overtreatment of malaria in children in a secondary healthcare centre in Sekondi-Takoradi, Ghana. Trop Dr. 2016;46:191-8.

14. Ndyomugyenyi R, Magnussen P, Lal S, Hansen K, Clarke SE. Appropriate targeting of artemisinin-based combination therapy by community health workers using malaria rapid diagnostic tests: findings from randomized trials in two contrasting areas of high and low malaria transmission in south-western Uganda. Trop Med Int Health. 2016:21:1157-70.

15. Bbosa F, Wesonga R, Jehopio P. Clinical malaria diagnosis: rule-based classification statistical prototype. Springerplus. 2016:5(939):6.

16. Chandramohan D, Carneiro I, Kavishwar A, Brugha R, Desai V, Greenwood B. A clinical algorithm for the diagnosis of malaria: results of an evaluation in an area of low endemicity. Trop Med Int Health. 2001;6:505-10.

17. Ferrão JL, Mendes JM, Painho M, João SZ. Spatio-temporal variation and socio-demographic characters of malaria in Chimoio municipality, Mozambique. Malar J. 2016;15:329.

18. Littrell M, Sow GD, Ngom A, Ba M, Mboup BM, Dieye Y, et al. Case investigation and reactive case detection for malaria elimination in northern Senegal. Malar J. 2013;12:331.

19. Lindblade KA, Steinhardt L, Samuels A, Kachur SP, Slutsker L. The silent threat: asymptomatic parasitemia and malaria transmission. Expert Rev Anti Infect Ther. 2013;11:623-39.

20. Ministère de la Santé, de la Prévention et de l'Hygiène Publique/PNLP. Bulletin Annuel 2014. Dakar, Senegal. 2015.

21. Ministère de la Santé, de la Prévention et de l'Hygiène Publique/PNLP. Bulletin Annuel 2015. Dakar, Senegal. 2016

\section{Submit your next manuscript to BioMed Central and we will help you at every step:}

- We accept pre-submission inquiries

- Our selector tool helps you to find the most relevant journal

- We provide round the clock customer support

- Convenient online submission

- Thorough peer review

- Inclusion in PubMed and all major indexing services

- Maximum visibility for your research

Submit your manuscript at www.biomedcentral com/submit
C Biomed Central 